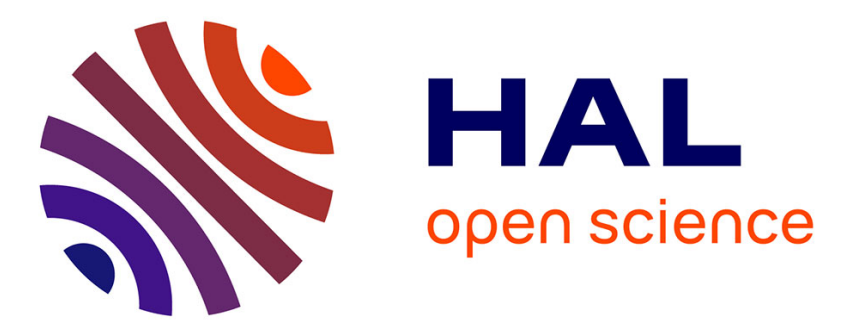

\title{
Nonlinear Equivalent-Circuit Modeling of a fast Photodiode
}

Zeina Abdallah, Alexandre Rumeau, Arnaud Fernandez, Gilles Cibiel, Olivier Llopis

\section{- To cite this version:}

Zeina Abdallah, Alexandre Rumeau, Arnaud Fernandez, Gilles Cibiel, Olivier Llopis. Nonlinear Equivalent-Circuit Modeling of a fast Photodiode. IEEE Photonics Technology Letters, 2014, 26 (18), pp.1041-1135. hal-01066679

\section{HAL Id: hal-01066679 https://hal.science/hal-01066679}

Submitted on 22 Sep 2014

HAL is a multi-disciplinary open access archive for the deposit and dissemination of scientific research documents, whether they are published or not. The documents may come from teaching and research institutions in France or abroad, or from public or private research centers.
L'archive ouverte pluridisciplinaire HAL, est destinée au dépôt et à la diffusion de documents scientifiques de niveau recherche, publiés ou non, émanant des établissements d'enseignement et de recherche français ou étrangers, des laboratoires publics ou privés. 


\title{
Nonlinear Equivalent-Circuit Modeling of a Fast Photodiode
}

\author{
Zeina Abdallah, Alexandre Rumeau, Arnaud Fernandez, Gilles Cibiel, Olivier Llopis
}

\begin{abstract}
A new nonlinear equivalent circuit model of a photodiode is proposed to describe the conversion of the laser relative intensity noise to microwave phase noise. This conversion is realized through a nonlinear $\mathrm{RC}$ circuit. The validity of this model has been confirmed by the good agreement between simulation and experimental results at different microwave frequencies. These simulations have been performed by using commercially available harmonic balance simulation software.
\end{abstract}

Index Terms-Equivalent circuits, Nonlinearities, Photodiode, Phase noise, Optoelectronic devices, Microwave oscillators.

\section{INTRODUCTION}

$\mathrm{R}$ F photonics technology offers an attractive alternative to classical electronic approach in various microwave systems. Fiber optics is indeed a small weight, low cost and small size technology for the transmission of microwave signals in a system. Moreover, the low losses and ultra-wide bandwidth in optical fibers added to the high quality factor of optical resonators allow interesting functionalities for microwave signal processing.

One specific application named optoelectronic oscillator (OEO) allows the generation of ultra-stable spectrally pure microwave reference frequencies when the microwave photonic link is used as a feedback loop [1]. However, the OEO performance is affected by the noise of optical and microwave components in the loop. Amplitude and phase noise generated in the loop will affect the noise of the output signal, and degrade its spectral purity. Furthermore, due to optical link's nonlinearities, laser's amplitude and phase noise can be converted to microwave noise. The photodiode (PD) seems to be the main device responsible for these conversions [2]. To quantify and investigate this last phenomenon, we founded relevant to apply a physical equivalent circuit model of photodiode in a microwave circuit simulator, such as Agilent ADS.

In previous works, several equivalent electrical circuit models have been proposed for fast photodiodes [3-6]. To name only a few, G. Wang et al. have developed a time-delay equivalent-circuit model which includes both the carrier transitinduced time-delay effect and stored charge effect of p-i-n photodiodes in high-power operation [3]. Recently, M. Piels et

This work is supported by CNES, the Regional Council of Midi Pyrénées, and the French MOD through DGA-ANR-11-ASTR-0029 MINOTOR contract.

Zeina Abdallah, Alexandre Rumeau, Arnaud Fernandez, and Olivier Llopis are with LAAS-CNRS, Toulouse University, UPS, Toulouse, France.

Zeina Abdallah and Gilles Cibiel are with CNES, Toulouse, France.

Contact: zeina.abdallah@laas.fr al. have presented a new method for the simulation of photodiode nonlinearities, with a model including the effects of non-uniform three dimensional absorption and self-heating [4]. However, none of the previously proposed models treated completely the nonlinear behavior of the photodiode which causes the conversion of the amplitude laser noise to RF phase noise. The lack of model regarding this topic made this research necessary.

In this letter, a new photodiode nonlinear equivalent circuit model is proposed. This model is able to describe the conversion of the laser relative intensity noise into microwave phase noise. It completes the modeling approach of microwave optical systems suggested by $\mathrm{H}$. Brahimi et al. [7], which was not able of fully describe this conversion, especially close to the carrier ( $1 / \mathrm{f}$ noise).

\section{CONVERSION OF AM LASER NOISE TO RF PHASE NOISE}

The noise performance of a microwave optical system depends on several parameters, such as the laser noise and photodiode noise. However, at high optical power, the signal to noise ratio is generally dominated by the laser amplitude noise, or RIN (relative intensity noise). Moreover, the low frequency components of the RIN can be converted to RF amplitude and phase noise through the devices nonlinearities, and especially the photodiode, as it has been reported in delay line based OEOs by Eliyahu et al. [2].

For low optical power levels, below the PD saturation power, the number of carriers in the PD increases with the increase of light power. Since the propagation speed of the RF signal depends on the number of carriers in the semiconductor, the RF phase will change as well [2].

Therefore, the laser amplitude fluctuations will transfer into RF phase fluctuations, in a way that can be described by the qualitative equation:

$$
S_{\Phi}(f)=R I N(f) \cdot P_{O p t}^{2} \cdot\left(d \Phi / d P_{o p t}\right)^{2}
$$

where $S_{\phi}(f)$ is the RF phase noise spectral density $\left(\mathrm{rad}^{2} / \mathrm{Hz}\right)$ at offset frequency $f$ from the RF carrier, and $\frac{d \Phi}{d P_{\text {Opt }}}$ is the RF phase-to-optical power slope ( $\mathrm{rad} / \mathrm{mW})$.

We have thus been particularly interested in the evolution of the $\frac{d \Phi}{d P_{\text {opt }}}$ parameter, both in our measurement and modelling approaches.

\section{EXPERIMENTAL PROCEDURES}

Our studies have started with the measurement of the RF power and the relative RF phase at the output of a Discovery DSC30S photodiode as a function of the modulated optical power at the input of the PD. 
Therefore, the experimental set-up (Fig. 1) has been realized with a $20 \mathrm{~mW}$ telecommunications laser module $(1550 \mathrm{~nm})$, temperature controlled and externally modulated at $10 \mathrm{GHz}$ by means of a Mach-Zehnder modulator (MZM) biased at $\mathrm{V}_{\pi / 2}$ in order to get a linear modulation (LM). The microwave signal comes from a calibrated vector network analyzer (VNA) working in a continuous wave (CW) mode. An Erbium Doped Fiber Amplifier (EDFA) has been also included in the set-up, in order to get a higher optical power on the PD. The amplitude and phase responses of the $S_{21}$ (transmission) parameter were accurately measured while changing the received optical power at the photodiode input. To this purpose, a variable optical attenuator (VOA) has been used just before the photodiode, and therefore the MZM nonlinearity versus the optical power can be discarded.

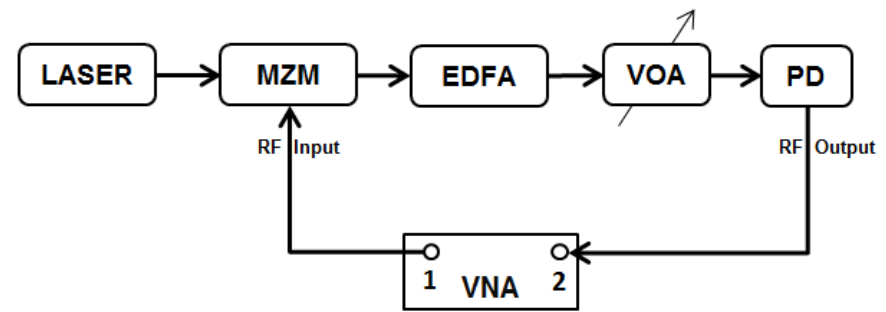

Fig. 1: Characterization bench of the photodiode's amplitude and phase response.

The experimental results, presented in Fig. 2, clearly show the nonlinear behavior of the PD and its ability to convert the laser RIN into RF phase noise. However, a zero slope in the photodiode's relative RF phase can be observed at optical input power of about $8-10 \mathrm{~mW}$. At this specific point, the conversion of the laser amplitude noise is null [2]. This zero slope is particularly interesting in the context of low microwave phase noise [8].The zero slope occurs at the beginning of the PD saturation. However, strong saturation on the RF amplitude is only visible at much higher power.

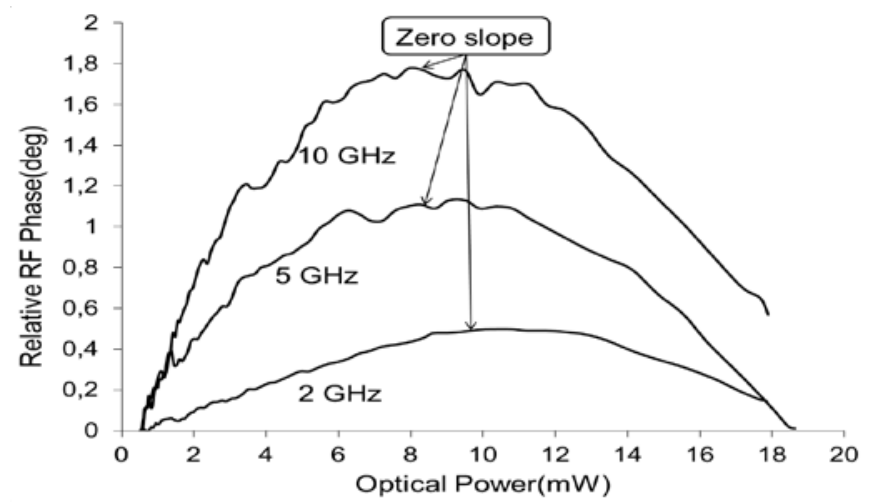

Fig. 2: Relative RF Phase of the Discovery photodiode, versus the received optical power, for $3 \mathrm{RF}$ frequencies.

Furthermore, the relative RF phase increases with the increase of the modulation frequency. Thus, this noise conversion phenomenon will be more critical when the application is at higher frequencies. It is noteworthy that this phase parameter also varies with the temperature fluctuations of the MZM, which change slightly its bias point. For this reason, we controlled the MZM temperature by fixing the MZM on a thick brass plate with foam insulation. Another alternative, which has also been used for some of our experiments, is to take benefit of an active MZM bias locking system (in our case, a Photline MBC-AN controller).

\section{THE PD NONLINEAR MODEL}

The nonlinear behavior of the photodiode could be also a result of a variation of its output capacitance, but after an experimental study of a high speed InGaAs/InP PIN photodiode chip, we have had the confirmation that this behavior only comes from the carrier transit-time. We have indeed taken advantage of the photodiode chip to measure the output reflection coefficient $S_{22}$ at several input optical powers using the same experimental bench described in the above section. Fig. 3 shows the measured real and imaginary parts of $\mathrm{S}_{22}$ versus frequency (from $40 \mathrm{MHz}$ to $20 \mathrm{GHz}$ ) for several optical powers. It is clearly visible that the $S_{22}$ is constant versus the optical power, and that the output capacitance is constant as well.

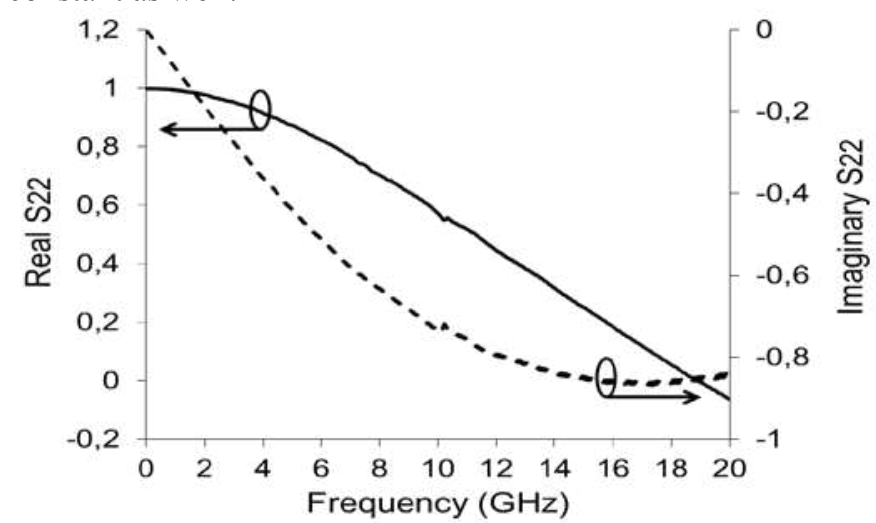

Fig. 3: Measured real (solid) and imaginary (dotted) parts of $S_{22}$ versus frequency of PIN PD chip for several optical powers.

Agilent ADS has been chosen to implement the PD model. The interest of this harmonic balance simulator lies in its ability to simulate the noise conversions between different carrier frequencies, even if these frequencies are as far apart as DC, $10 \mathrm{GHz}$ (microwave signal) and $200 \mathrm{THz}$ (optical carrier). It is however noteworthy that Agilent ADS library does not include optical or optoelectronic devices. These components have been implemented using electrical equivalent models (frequency sources, delay lines...) or mathematical equations thanks to the symbolically defined devices module of ADS (case of the MZM modulator). Details on this approach can be found in [7].

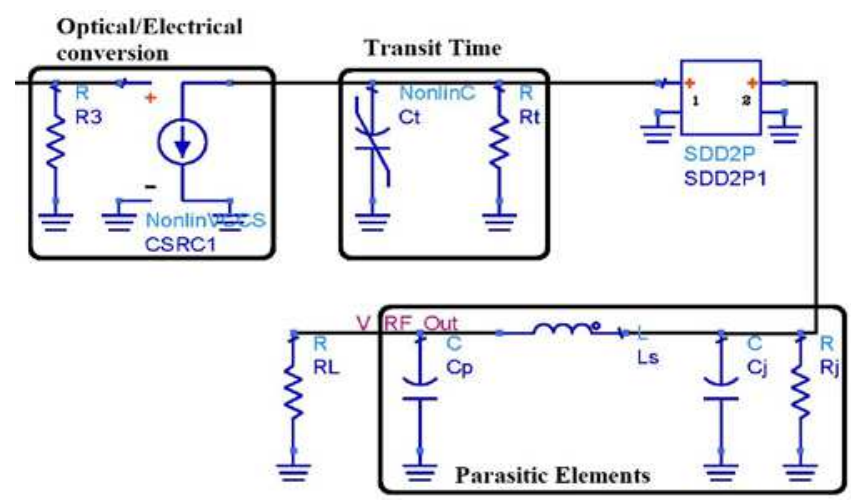

Fig. 4: A nonlinear equivalent circuit of a photodiode. 
The proposed nonlinear equivalent circuit of the photodiode is presented in Fig. 4. The Optical/Electrical conversion is described as a quadratic detector using a nonlinear voltage controlled current source. The center section deals with the carrier transit time effects, which is represented by an $\mathrm{R}_{t} \mathrm{C}_{t}$ circuit, where the capacitance is a nonlinear function of voltage. The nonlinear capacitance is defined as:

$$
C_{t}(V)=\alpha_{1}+\alpha_{2} \cdot V+\alpha_{3} \cdot V^{2}
$$

where $\mathrm{V}$ is the voltage across the capacitance, and $\alpha_{1}, \alpha_{2}$, and $\alpha_{3}$ are the polynomial coefficients.

This nonlinear time-delay equivalent circuit is separated from the parasitic elements by a nonlinear isolator which also allows a current compression for high optical power. This isolator is defined as:

$$
I(V)=I s a t \cdot \tanh \left(\frac{V}{R \cdot I s a t}\right)
$$

where I and $\mathrm{V}$ are the output current and input voltage of the isolator, Isat is the current saturation and its value is based on experimental studies.

Finally, the parasitic parameters were added to the model, where $R_{j}$ and $C_{j}$ are the junction resistance and capacitance, respectively. $\mathrm{C}_{\mathrm{p}}$ is the parasitic capacitance, and $\mathrm{L}_{\mathrm{s}}$ is the series inductance.

\section{RESULTS AND DISCUSSION}

In Fig. 5, the comparison between the measured and simulated relative RF phase data of the Discovery PD are presented for three different RF frequencies $(2,5$, and 10 $\mathrm{GHz})$.

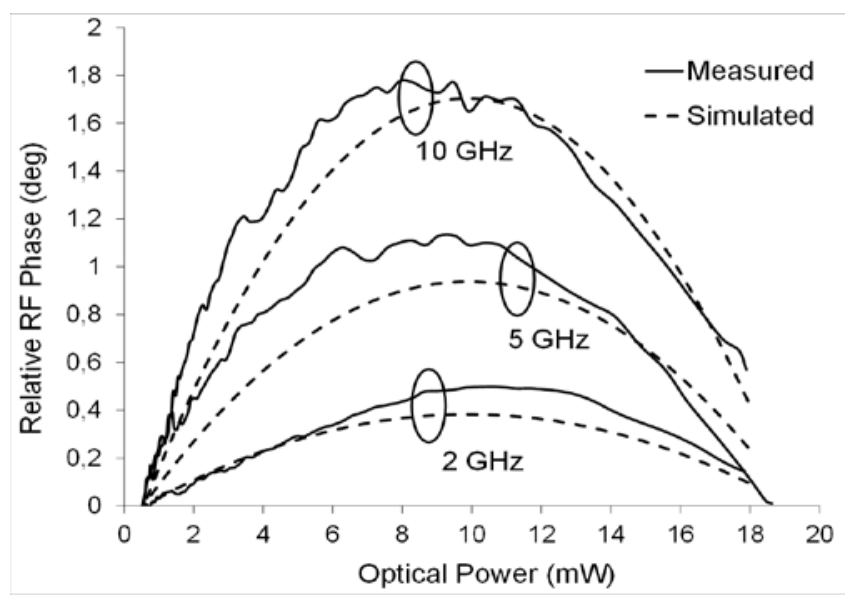

Fig. 5: Simulated (dotted) and experimental (solid) Relative RF phase of the Discovery PD versus input optical power, for three different frequencies.

By carefully choosing the values for the carrier transit-time components $\left(\mathrm{R}_{\mathrm{t}}, \mathrm{C}_{\mathrm{t}}\right)$, and especially the polynomial coefficients $\left(\alpha_{1}, \alpha_{2}\right.$ et $\left.\alpha_{3}\right)$ of the nonlinear capacitance $C_{t}$, the simulated relative RF phase results are in good agreement with the measured results, with a maximal error level in the relative phase difference below $0.3^{\circ}$.

Taking into account the difficulties and the precision of the measurements (particularly because of their temperature sensibility), we believe that the model presented in this letter is successful at modeling the photodiode nonlinear behavior. The simulated and measured frequency response of the photodiode at various optical power levels are depicted in Fig.6. Again, a relatively good agreement is found between simulated and measured data.

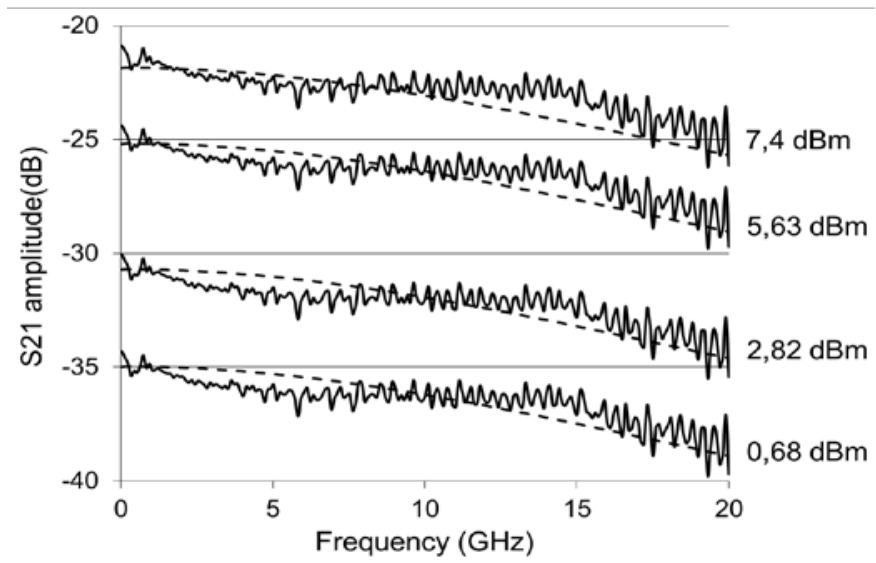

Fig. 6: Simulated (dotted) and experimental (solid) results of $S_{21} R F$ amplitude of the Discovery PD versus frequency for several optical powers.

\section{CONCLUSION}

We have proposed a nonlinear equivalent-circuit model of a photodiode which takes into account the carrier transit time delay. This model is skillful when it comes to represent the conversion of the laser amplitude noise into RF phase noise. We have been able to get a good match with the experimental data for the RF phase to optical power sensitivity factor with this model. Further investigations are in progress, particularly to simulate the $1 / \mathrm{f}$ phase noise in complex microwave optical systems using this approach.

\section{REFERENCES}

[1] X. S. Yao and L. Maleki, "Optoelectronic microwave oscillator", J. Opt. Soc. Am. B, vol. 13, No. 8, August 1996, pp. 1725-1735.

[2] D. Eliyahu, D. Seidel, L. Maleki, "RF Amplitude and Phase-Noise Reduction of an Optical Link and an Opto-Electronic Oscillator", IEEE Trans. on Microwave Theory and Tech., vol. 56, n 2, Feb 2008, pp. 449456.

[3] G. Wang, T. Tokumitsu, I. Hanawa, Y. Yoneda, K. Sato, M. Kobayashi, "A time-Delay Equivalent-Circuit Model of Ultrafast p-i-n Photodiodes", IEEE Trans. on Microwave Theory and Techniques, vol. 51, no. 4, April 2003, pp. 1227-1233.

[4] M. Piels, A. Ramaswamy, and J. E. Bowers, "Nonlinear modeling of waveguide photodetectors," Optics Express, vol. 21, no. 13, pp. 15634$15644,2013$.

[5] H. Jiang and P. K. L. Yu, "Equivalent Circuit Analysis of Harmonic Distortions in Photodiode", IEEE Photonics Technology Letters, vol. 10, $\mathrm{n}^{\circ} 11$, November 1998, pp. 1608-1610.

[6] M. Nikoufard, X.J.M. Leijtens, Y.C. Zhu, T.J.J. Kwaspen, E.A.J.M Bente, F.H. Groen, and M.K. Smit, "Modelling and Characterization of InP-Based high-speed pin-photodiode", Proceedings Symposium IEEE/LEOS Benelux Chapter, 2003, Enschede.

[7] H. Brahimi, H.L. Martinez-Reyes, P.H. Merrer, A. Bouchier, O. Llopis, "A CAD approach of Microwave Optical Systems including Noise performance", Proc. of the 2009 European Microwave Conf. (EuMC), Rome, Italy, 2009, pp. 1642-1645.

[8] W. Zhang, T. Li, M. Lours, S. Seidelin, G. Santarelli, and Y. Le Coq, "Amplitude to phase conversion of InGaAs pin photo-diodes for femtosecond lasers microwave signal generation", Appl. Phys. B, vol. 106, issue 2, Feb. 2012, pp. 301-308. 